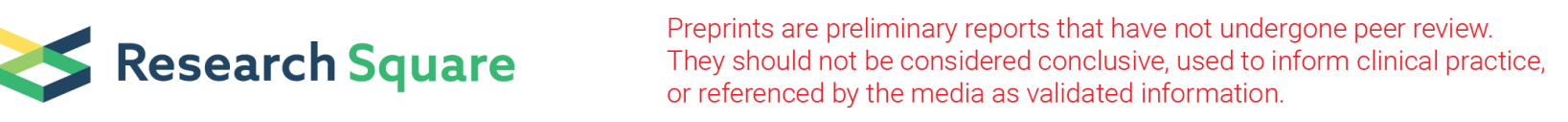

\title{
Hematoma Cavity Separation and Neomembrane Thickness are Potential Triggers of Recurrence of Chronic Subdural Hematoma
}

Hongbin Liu

The First People's Hospital of Ziyang

Rudan Yan

The First People's Hospital of Ziyang

Fei Xie (D349632449@qq.com)

The First People's Hospital of Ziyang

Seidu A. Richard

Princefield University

\section{Research Article}

Keywords: CSDH, Craniotomy, Craniotomy, Connector, Endoscopy.

Posted Date: February 21st, 2022

DOI: https://doi.org/10.21203/rs.3.rs-1371222/v1

License: (c) (i) This work is licensed under a Creative Commons Attribution 4.0 International License. Read Full License 


\section{Abstract}

Background: Chronic subdural hematoma (CSDH) is the anomalous accumulation of liquified blood in the subdural space usually after a head injury in the elderly. Almost all the research on surgical techniques and endoscopic assisted evacuation of CSDH focused on the just the evacuation and not abnormal anatomical structures that causes recurrences.

Objectives: We investigated abnormal anatomical structures that triggers recurrence of CSDH during craniotomy as well as burr-hole craniostomy with endoscopic assistance.

Materials and Method: We retrospectively analyzed all patients with CSDH who underwent craniostomy and burr-hole craniotomy with endoscopic assisted evacuation of hematoma between April 2017 and November 2020 at our institution. Clinical data obtained was categorized into patient-related, radiology as well as surgery and endoscopic evaluations.

Results: A total of 143 patients (109 men and 34 women) aged 43-94 years (mean age, 68.35 years) with CSDH were included in this study. We observed a recurrence rate of $4.9 \%(7 / 143)$. Recurrences occurred between 2-6 months after the operation in patients with recurrences.

Our data revealed that, age, hypertension, history of injury, diabetes, antiplatelet or anticoagulant use were not associated with hematoma recurrence. Nevertheless, all the patients with recurrence of hematoma were males. Interestingly, our univariate and multivariate analyses found neomembrane thickness and hematoma cavity separation as independent risk factors $(\mathrm{OR}, 45.822 ; 95 \% \mathrm{Cl}, 2.666-787.711 ; \mathrm{p}=0.008)$ for the recurrence of $\mathrm{CSDH}(\mathrm{p}<0.05)$. Also, we observed thickened membranes connecting/separating the dura and the thickened arachnoid/pia matters in all the 7 patients with hematoma recurrence.

Conclusion: The treatment of patients with CSDH ought to include the identification and resection of abnormal thickened membranes connecting/separating the dura and the thickened arachnoid/pia matters to avoid recurrence. Comparatively, endoscopy showed hematoma cavity separation or neomembrane thickness just as seen during craniotomy.

\section{Introduction}

Chronic subdural hematoma (CSDH) is the anomalous accumulation of liquified blood in the subdural space usually after a head injury in the elderly[1-4]. Most often, the accumulated fluid exerts pressure on the brain tissues resulting in neurologic complications[2, 3]. Initially, CSDH was perceived as a gradual as well as recurring hemorrhage caused by ruptured cortical bridging veins during the trauma[4-6]. Currently, multiple factors such as inflammation, angiogenesis, recurrent micro-hemorrhages, as well as local coagulopathy in the subdural space has been implicated as the cause of the $\operatorname{CSDH}[5,7,8]$. Noteworthily, inflammatory reactions often trigger the formation of hematoma, re-bleeding as well as maintenance and liquification of the hematoma[5, 7, 8]. 
Clinically, the patients often present with symptoms such as headaches, behavioral changes, focal deficits, seizures as well as hemiparesis[9, 10]. Non-contrast Computed tomographic (CT) scan is usually the gold-standard radiology modality use in detecting this anomalous liquified blood in subdural space[5]. Craniotomy, burr-hole craniostomy as well as twist-drill craniostomy, are the well-known surgical techniques for the treatment of $\operatorname{CSDH}[1,11-13]$. Nevertheless, about $2.5-33 \%$ of recurrence rates are associated with the treatment modalities above[5, 14, 15].

Endoscope-assisted evacuation of CSDHs is an accepted technique, though not generally used[16-23]. It is an amalgamation of burr-hole evacuation of $\mathrm{CSDH}$ as well as inspection of the subdural space with an endoscope[11]. This technique is advantageous because, the placement of the catheter is often under visual control, detection of septations as well as timely recognition of essential cortexes or vessel injury during surgery[11]. Almost all the research on surgical techniques and endoscopic assisted evacuation of CSDH focused on the just the evacuation and not abnormal anatomical structures that causes recurrences. Thus, our study explores factors that triggers recurrence of CSDH during and after treatment.

\section{Materials And Method}

\section{Patients}

We retrospectively analyzed all patients with CSDH who underwent craniotomy and burr-hole craniostomy with endoscopic assisted evacuation of hematoma at the Department of Neurosurgery, $\mathrm{Zi}$ Yang first people's hospital, China, between April 2017 and November 2020. This study was reviewed and approved by our Institutional Research Review Board. The patients as well as their relatives were dually informed about our intention to involve them in a study and they fully concerted to the use of their information. All the patients and their relatives signed written informed consents. Factors triggering recurrence of CSDH were divided into three broad categories such as patient comorbid factors, hematoma subtypes on radiology as well as abnormal anatomical structures. Clinical data obtained was categorized into patient-related, radiology as well as surgery and endoscopic evaluation. Patient-related data such as age, sex, history of injury, hypertension, diabetes, antiplatelet or anticoagulant use were obtained and documented at time of admission in the ward. All the patients were referred to our hospital from periphery hospitals with history of CSDH.

\section{Radiological Evaluation}

The diagnosis of CSDH patients was re-established by CT scan on admission. Radiology related data such as unilateral or bilateral hematoma, hematoma thickness, density of the hematoma, midline brain shift and brain atrophy were obtained and documented. Also, initial detection of hematoma cavity separations and neomembrane thicknesses were established on CT scan. Neomembrane thicknesses were seen as thickened dura structures while hematoma cavity separations were seen as two or more subdural cavities. All hematomas were classified into four types according to their density on CT scan: 
Low-density (<25 Hounsfield unit [HU]), iso-density (25-35 HU), hyper-density (> $35 \mathrm{HU})$, and mixeddensity[24]. Brain atrophy was classified into the three types based on CT findings: no or mild atrophy, moderate atrophy, such as dilated sulci, and severe atrophy, such as widely dilated sulci and subdural space[25]. Additionally, postoperative CT scans were performed within 3 days after surgery.

\section{Surgical Management}

All patients who underwent surgery were identified as CSDH before surgery. Patients either underwent a single burr-hole craniostomy with endoscopic assisted hematoma evaluation or craniotomy under general anesthesia and closed-system drainage. All patients were placed in lateral position during the operation and after removing the bone flap, the hematoma was flushed out via irrigation with warm physiological saline solution to reduce postoperative gas accumulation and residual of the hematoma. Neomembrane thicknesses, hematoma cavity separations as well as all other radiological parameters were reestablished intraoperatively. Intraoperation, neomembrane thicknesses were seen as thickened dura structures relative to the normal dura while hematoma cavity separations were seen as two or more subdural cavities. Also, patients were observed under flexible endoscopy for the presence of a hematoma cavity and the formation as well as neomembrane thickness in the single burr-hole craniostomy treated patients. In all patients, drainage catheters were removed 48 hours whether or not residual hematomas were seen on postoperative CT scans after the operation.

\section{Followed-up And Evaluation Of Recurrence}

All patients were followed monthly for 6 months after surgery at outpatient to establish recurrence of CSDH. CT scan were performed in all the patient's whether they present with neurological symptoms or not to establish recurrence during follow-ups. Recurrence was defined as any increase in the volume of ipsilateral subdural hematoma on CT scan after removal of drainage system. After the first 6 months, all the patients were subsequently followed for at least 2 years. We had no missing data and no patients were lost on follow-ups.

\section{Statistical analysis}

We used commercially available software (IBM SPSS Statistics for Windows, Version 25.0; IBM Corp, Armonk, New York, USA) for statistical analyses. Chi-square test or Fisher's exact test was used for univariate analyses. In logistic regression analysis, adjustments were made for age, gender, diabetes, hypertension, antiplatelet and/or anticoagulant use, history of trauma, bilateral hematoma, brain atrophy, hematoma thickness and midline shifting. Model covariates were determined by descriptive statistics and previous studies. The relationship of each predictive factor and CSDH recurrence was presented as odds ratio (OR) and $95 \%$ confidence interval $(\mathrm{Cl})$. A p-value $<0.05$ was defined as statistically significant.

\section{Results}




\section{Patients Demographic Characteristics}

A total of 143 patients ( 109 men and 34 women) aged 43-94 years (mean age, 68.35 years) with CSDH were included in this study. The baseline characteristics are described in Table 1. Among them, 76 patients admitted haven experienced one or more episodes of apparent head trauma while 67 could not remember traumatic events. The time interval between trauma and the first operation was $10 \sim 90$ days (mean 24 days). Six patients where on aspirin, seven patients were on clopidogrel and four patients were on warfarin. Our data revealed that, age, hypertension, history of injury, diabetes, antiplatelet or anticoagulant use were not associated with hematoma recurrence (Table 1\&2). Nevertheless, all the patients with recurrence of hematoma were males. 
Table 1

Summary of baseline characteristics in no recurrence group and recurrence group in 143 patients with $\mathrm{CSDH}$

\begin{tabular}{|c|c|c|c|}
\hline Factor & No Recurrence (\%) & Recurrence (\%) & $P$ value \\
\hline Total & $136(95.1 \%)$ & $7(4.9 \%)$ & \\
\hline Sex & & & 0.231 \\
\hline Female & $34(23.8 \%)$ & $0(0.0 \%)$ & \\
\hline Male & $102(71.2 \%)$ & $7(4.9 \%)$ & \\
\hline Age (years) & $70.75 \pm 8.91$ & $64.14 \pm 15.52$ & 0.418 \\
\hline Diabetes & $22(15.4 \%)$ & $2(1.4 \%)$ & 0.736 \\
\hline Hypertension & $60(41.9 \%)$ & $1(0.7 \%)$ & 0.244 \\
\hline Antiplatelet and/or anticoagulant use & $16(11.2 \%)$ & $1(0.7 \%)$ & 0.436 \\
\hline Bilateral hematoma & $16(11.2 \%)$ & $0(0.0 \%)$ & 1.000 \\
\hline History of trauma & $73(51.0 \%)$ & $3(2.1 \%)$ & 0.949 \\
\hline Brain atrophy & & & 0.758 \\
\hline Mild & $102(71.3 \%)$ & $5(3.5 \%)$ & \\
\hline Moderate & $26(18.2 \%)$ & $2(1.4 \%)$ & \\
\hline Severe & $8(5.6 \%)$ & $0(0 \%)$ & \\
\hline Hematoma thickness $(\mathrm{cm})$ & $1.97 \pm 0.56$ & $2.13 \pm 0.52$ & 0.482 \\
\hline Midline shift (cm) & $0.86 \pm 0.55$ & $0.69 \pm 0.51$ & 0.426 \\
\hline Density of hematoma on CT & & & $\varangle 0.001$ \\
\hline Isodensity hematoma & $64(44.8 \%)$ & $0(0 \%)$ & \\
\hline Low-density hematoma & $26(18.2 \%)$ & $0(0 \%)$ & \\
\hline High-density hematoma & $23(16.1 \%)$ & $1(0.7 \%)$ & \\
\hline Mixed density hematoma & $23(16.1 \%)$ & $6(4.2 \%)$ & \\
\hline Neomembrane in CT & $13(9.1 \%)$ & $4(2.8 \%)$ & 0.003 \\
\hline Hematoma cavity separation in CT & $11(7.7 \%)$ & $4(2.8 \%)$ & 0.002 \\
\hline $\begin{array}{l}\text { Hematoma cavity separation or } \\
\text { Neomembrane in endoscopy }\end{array}$ & $34(23.8 \%)$ & $6(4.2 \%)$ & 0.001 \\
\hline $\begin{array}{l}\text { Abbreviations: CSDH: Chronic subdura } \\
\text { centimeter. }\end{array}$ & oma, CT: Computec & graphy, \%: per & ge, CM: \\
\hline
\end{tabular}


Table 2

Logistic regression analysis of predictive factors for recurrence of CSDH

\section{$P$ value $\quad O R(95 \% \mathrm{Cl})$}

\section{Model 1}

Gender

$0.998 \quad N A$

Age(years)

0.362

0.949(0.847-1.063)

Hypertension

0.085

$0.060(0.002-1.474)$

Diabetes

0.514

$0.419(0.031-5.718)$

Antiplatelet and/or anticoagulant use

0.101

14.836(0.590-372.953)

Bilateral hematoma

0.998

NA

History of trauma

0.861

$0.839(0.117-6.008)$

Mild brain atrophy

0.320

1

Moderate brain atrophy

0.131

$10.816(0.491-238.388)$

Severe brain atrophy

0.999

NA

Hematoma thickness

0.313

2.885(0.368-22.592)

Midline shift

0.809

$0.748(0.071-7.850)$

Neomembrane in CT*

0.019

56.135(1.916-1644.653)

Model 2

Gender

0.998

NA

Age(years)

0.551

0.963(0.851-1.090)

Hypertension

0.162

0.109(0.005-2.441)

Diabetes

0.572

0.488(0.040-5.905)

Antiplatelet and/or anticoagulant use

0.099

14.848(0.603-347.929)

History of trauma

0.891

0.872(0.125-6.088)

Bilateral hematoma

0.998

NA

Mild brain atrophy

0.528

1

Moderate brain atrophy

0.259

6.586(0.250-173.249)

Severe brain atrophy

0.999

NA

Abbreviations: OR: Odds ratio; Cl: Confidence interval; NA: Not Available, CSDH: Chronic subdural hematoma; CT: Computed tomography; *: Significant difference. 


\begin{tabular}{|lll|}
\hline & P value & OR $(95 \%$ Cl $)$ \\
\hline Hematoma thickness & 0.347 & $2.715(0.339-21.774)$ \\
\hline Midline shift & 0.586 & $0.498(0.040-6.125)$ \\
\hline Hematoma cavity separation in CT* & 0.019 & $40.173(1.833-880.678)$ \\
\hline Model 3 & & \\
\hline Gender & 0.997 & NA \\
\hline Age(years) & 0.266 & $0.914(0.780-1.071)$ \\
\hline Hypertension & 0.161 & $0.114(0.005-2.373)$ \\
\hline Diabetes & 0.797 & $1.409(0.104-19.141)$ \\
\hline Antiplatelet and/or anticoagulant use & 0.869 & $1.285(0.065-25.561)$ \\
\hline Bilateral hematoma & 0.998 & NA \\
\hline History of trauma & 0.451 & $2.376(0.251-22.522)$ \\
\hline Mild brain atrophy & 0.794 & 1 \\
\hline Moderate brain atrophy & 0.498 & $3.968(0.074-212.904)$ \\
\hline Severe brain atrophy & 0.999 & NA \\
\hline Hematoma thickness & 0.704 & $1.593(0.144-17.632)$ \\
\hline Midline shift & 0.446 & $0.287(0.012-7.131)$ \\
\hline Hematoma cavity separation or* & 0.008 & $45.822(2.666-787.711)$ \\
\hline Neomembrane during endoscopy & & \\
\hline $\begin{array}{l}\text { Abbreviations: OR: Odds ratio; Cl: Confidence interval; NA: Not Available, CSDH: Chronic subdural } \\
\text { hematoma; CT: Computed tomography; : Significant difference. }\end{array}$ & \\
\hline
\end{tabular}

\section{Radiological Outcomes}

CT scan at admission showed that, the CSDH was left-sided in 75 patients, right-sided in 52 patients, and bilateral in 16 patients. We observed mixed density hematoma in 29 patients, iso-density hematoma in 64, high-density hematoma in 24, low-density hematoma in 26. CSDH was observed in 107 patients with mild brain atrophy, 28 patients with moderate brain atrophy and 8 patients with severe brain atrophy. We observed midline shifts in 123 patients. While 20 patients had no midline shifts on CT scan. Midline shift ranged from 0-2.2 cm (mean $0.85 \mathrm{~cm}$ ). Hematoma thickness ranged from 8-33 cm (mean $1.98 \mathrm{~cm}$ ). Hematoma cavity separation was found in 15 patients, and neomembrane thicknesses were detected in 17 patients. Out of 17 patients with neomembrane thickness, mixed density hematoma was observed in 
11 patients, iso-density hematoma in 2, high-density hematoma in 1 and low-density hematoma in 3. Also, all the 15 patients with hematoma cavity separation showed mixed density hematoma. CT characteristic of patients are as shown in (Fig. 1A-C). Also, characteristic or radiological outcomes is as shown in Table 1. Furthermore, unilateral or bilateral hematoma, hematoma thickness as well as midline shift were not associate with hematoma recurrence.

\section{Surgical And Endoscopic Outcomes}

Total of 133 patients underwent single burr-hole craniostomy with endoscopic assisted hematoma evacuations and closed drainage while 10 patients underwent craniotomy (Fig. 2A). We resected the thickened neomembrane in two patients after craniotomy (Fig. 2B\&C) and none of them experienced recurrence of the hematoma. Intraoperatively, we observed thickened membrane connecting/separating the dura and the thickened cortical surface comprising of arachnoid/pia matters (Fig. 2B\&C) in the two patients above which were resected. Postoperative CT scan showed total evaluation of the hematomas (Fig. 2D). None of patients developed surgical site complications after burr hole drainage.

Hematoma cavity as well as the formation of neomembranes and their thicknesses were visible on endoscopy in the single burr-hole craniostomy treated patients (Fig. 3A\&B). Quantitively, endoscopy showed hematoma cavity separation or neomembrane thickness in 40 patients. Comparatively, endoscopy showed hematoma cavity separation or neomembrane thickness just as seen during craniotomy (Fig. 2B\&C and Fig. 3A\&B). Similarly, on endoscopy, we observed the same thickened membranes connecting/separating the dura and the arachnoid/pie matter (Fig. 3B). These thickened membranes connecting/separating the dura and the thickened arachnoid/pia matters were often resected during endoscopy.

\section{Hematoma Recurrence And Patients' Outcomes After Surgery}

We did not observe recurrence within the first 3 days after operation during the hospitalization in all patients. We observed recurrence between 2-6 months after the operation in patients with recurrences. Overall, we observed recurrence of hematoma in 7 patients. Thus, the recurrence rate was $4.9 \%$ (7 of 143 ). Out of the 40 patients in whom endoscopy showed hematoma cavity separation or neomembrane thickness above, hematoma recurred in 6 of them.

One patient out of the 10 patients who underwent craniotomy had recurrence of the hematoma. Out of the 7 patients with hematoma recurrence, we observed mixed density hematoma in 6 patients during CT evaluation while one patient had high-density hematoma during CT scan evaluation (Table 1). Also, in all the 7 patients with hematoma recurrence, we observed neomembrane thickness as well as hematoma cavity separation on CT scan. Furthermore, we observed thickened membranes connecting/separating the dura and the thickened arachnoid/pia matters in all the 7 patients with hematoma recurrence (Fig. $2 \mathrm{C}$ 
\& 3B). We postulate that, inadequate or non-separation of thickened membranes connecting/separating the dura and the thickened arachnoid/pia matters could be a cause of the recurrence of hematoma. Thus, during the treatment of patients with $\mathrm{CSDH}$, these membranes ought to be identified and resected.

Univariate and multivariate analyses found neomembrane thickness and hematoma cavity separation as independent risk factors $(\mathrm{OR}, 45.822 ; 95 \% \mathrm{Cl}, 2.666-787.711 ; \mathrm{p}=0.008)$ for the recurrence of CSDH $(p<$ 0.05 ) (Table 1 \&2). Two patients died after the first six-months follow-ups. One died of pneumonia while the cause of the death of the other was unknown. Thus, the mortality rate was $1.39 \%$ (2 of 143 ). No patient was lost on follow-ups. Patients with recurrence were re-treated via burr-hole craniostomy and endoscopic assisted hematoma evacuation with closed-system drainage with more focus on the resection of thickened membranes connecting/separating the dura and the thickened arachnoid/pia matters. We did not observe further or second recurrence during 2 years follow-ups.

\section{Discussion}

$\mathrm{CSDH}$ is one of the most common post traumatic disorder managed by neurosurgeons $[3,26]$. It is estimated that, about 8.2-14.1 per 100,000 persons often develop this disease per years in the general population $[11,27,28]$. CSDH is typically associated with good recovery after treatment although about $2.5-33 \%$ of patients present with recurrence necessitating re-operation[5, 26]. Also, repeated recurrences as well as refractory CSDH although rare, have been reported[26]. Thus, our study explores factors that triggers recurrence of CSDH during and after treatment. Factors triggering recurrence of CSDH were divided into three broad categories such as patient comorbid factors, hematoma subtypes on radiology as well as abnormal anatomical structures. We observed a recurrence rate of $4.9 \%$. Recurrences occurred between 2-6 months after the operation in patients with recurrences.

Patient comorbid factors includes, advanced age, diabetes, hypertension, intracranial hypotension, alcohol consumption as well as antiplatelet or anticoagulant usage $[1,11,27,28]$. Also, radiological risk factors associated with the recurrence includes, uni- or bilateral hematoma, preoperative hematoma thickness and midline shift, hematoma density and internal architecture, cerebral atrophy, as well as hematoma volume[5, 29-32]. Furthermore, procedural risk factors such as surgical technique, hemorrhage, postoperative posture, postoperative subdural accumulation of gas has also been implicated as causes of recurrence of $\operatorname{CSDH}[5,11,27,28]$. Interestedly, our univariate and multivariate analyses revealed that, age, hypertension, history of injury, diabetes, antiplatelet or anticoagulant use, were not associated with hematoma recurrence. Nevertheless, all the patients with recurrence of hematoma were males.

CT scan is the gold standard diagnostic modality for evaluating patients with CSDH. The evaluation of CT predictors is of great extra significance subsequent to other recognized clinical predictors[5, 30, 32]. In our study, CT scan was capable of detecting of hematoma cavity separations and neomembrane thicknesses. Neomembrane thicknesses were seen as abnormal structures relative to the normal dura while hematoma cavity separations were seen as two or more subdural cavities. Miah et al with a meta- 
analysis observed that, hyperdense as well as mixed density hematoma were more linked to CSDH recurrence, as were laminar as well as separated architecture hematomas[5]. Furthermore, they indicated that, CSDH with high degree of hematoma thickness as well as midlines shift had more changes of recurrence[5]. They also found homogeneous hyperdense as well as mixed density hematoma to be more linked to recurrence rates[5].

Brain atrophy was observed in all the patients with CSDH. Most of the patients who developed CSDH had mild brain atrophy (107), 28 patients had moderate brain atrophy while 8 patients had severe brain atrophy. Recurrence occurred in 5 patients with mild brain atrophy and two patients with moderate brain atrophy. In our study, midline shift ranged from 0-2.2 cm (mean $0.85 \mathrm{~cm}$ ). Nevertheless, midline shift was not associated with hematoma recurrence according to our analysis. Also, unilateral or bilateral hematoma in our CSDH patients were not associate with recurrence of the hematoma. Moreover, hematoma thickness ranged from 8-33 cm (mean $1.98 \mathrm{~cm}$ ). Nevertheless, hematoma thickness was not also associated with recurrence of $\mathrm{CSDH}$ according to our analysis.

Iso-dense hematoma was the most prominent hematoma subtype according to our analysis followed by low-density hematoma and mixed density hematoma as well as high-density hematomas. Six patients with mix density hematoma had recurrence of the hematoma after treatment while one patient with highdensity hematomas had a recurrence of the hematoma after treatment. We did not observe hematoma recurrence in patients with iso-dense hematomas and low-density hematomas.

Noteworthily, interplay of inflammatory cytokines is the widely accepted phenomenon causing recurrence of $\operatorname{CSDH}[5,33-36]$. Traumatic rupture of the arachnoid membrane often results in mild hemorrhage from bridging veins as well as brain contusion[1,37,38]. Subsequently, cerebrospinal fluid gradually leaks into the collected blood via torn arachnoid membranes[1,37]. The accumulated mix fluid triggers an inflammatory reaction in the subdural space[5,33,34]. This inflammatory process involves the release of cytokines as well as several other inflammatory markers which maintains the hematoma as well as rebleeding tendencies[5,34-36]. This process leads to the formation of a primary neomembrane with rich vasculature.

The neomembrane usually consists of a thick outer neomembrane as well as a thin neomembrane surrounding the blood which also subsequently liquidifies[1,37]. The neomembrane, restrict the brain tissue from expanding resulting in subdural hematoma cavity that persists resulting in recurrence of the $\operatorname{CSDH}[1,37]$. We observed thickened membranes connecting/separating the dura and the thickened arachnoid/pia matters which sustains the separation of the hematoma cavities leading to recurrences of the hematomas after treatments.

Craniotomy, burr-hole craniostomy as well as twist-drill craniostomy are the widely used

surgical treatment modalities for $\mathrm{CSDH}[11-13]$. Currently, craniotomy under general anesthesia is mostly performed in organized hematomas or multilobulated as well as for recurrent incidents[11, 39]. Craniotomy is advantageous because, with a better exposure the surgeon is able to resect the outer 
capsule as well as neomembranes and also secure precise hemostasis[11, 39]. Although this surgical technique has the highest morbidity rate because of its invasiveness, it has the lowest recurrence rate compare to the other surgical modalities[11, 13,39].

In our study, ten patients underwent craniotomy. Intraoperatively, we observed a thickened membrane connecting/separating the dura and the thickened cortical surface comprising of arachnoid/pia matters in the two patients which we resected. Twist-drill craniostomy is mostly carried out at bedside under local anesthesia and amongst the three surgical techniques, it is the least invasive technique[11, 39]. Nevertheless, with this technique, profuse irrigation with saline, which appears to be advantageous in relation to the recurrence rate is often not achievable[11,39, 40]. Burr-hole craniostomy is performed under either general or local anesthesia and it is usually the first-line treatment modality[11, 12]. Nakaguchi et al observed that frontal insertion of a draining catheter tip decreases recurrence of the hematoma[19].

Chihara et al observed that, with the hematoma subtype, organized or septated CSDH frequently trigger recurrence of $\mathrm{CSDH}[41]$. In cases of septated $\mathrm{CSDH}$, a second burr-hole with robust as well as adequate irrigation often gives a good outcome in the second operation[26, 41]. Internal architecture types have also used for the categorization of CSDHs[5]. Nakaguchi et al classified CSDHs into homogeneous, laminar, separated as well as trabecular, based on anticipated phases in the natural history of a $\mathrm{CSDH}[42]$. Miah et al found a higher recurrence risk in laminar as well as separated hematoma compared to others hematomas[5].

We observed hematoma cavity separation in 15 patients, and neomembrane thickness in 17 patients. Our univariate and multivariate analyses revealed that, neomembrane thickness and hematoma cavity separation were independent risk factors for recurrence of CSDH. Although organized or septated and separation of membranes in CSDH have reported as trigger of recurrence[5, 18, 26, 41, 42], no study reported a thickened membrane connector/separator between the dura and the thickened arachnoid/pia matters as cause of recurrence of the CSDH. We observed thickened membranes connecting/separating the dura and the thickened arachnoid/pia matters in all the 7 patients with hematoma recurrence.

Successful evacuation of subdural haematomas, pus as well as hygromas have been achieved with the use of endoscopic $[16,43,44]$. The endoscope assists in the assessment of the subdural space to ensure satisfactory clot evacuation as well as haemostasis, and to incise abnormal membranes as well as trabecula in instances of loculated CSDHs[16, 23, 43, 44]. Furthermore, during the procedure, the surgeon is able to irrigate the hematoma cavity, insert a drainage tube toward the frontal convexity to evacuate subdural air, avoid cortical laceration that often happen as result of blind manipulation, coagulate the source of bleeding as well as separate the inner membrane carefully to aid in brain expansion[17-21]. Nevertheless, during the procedure, the endoscope may cause injury to the brain cortex[11].

We performed single burr-hole craniostomy with endoscopic assisted hematoma evaluation and closedsystem drainage in 40 patients. Hematoma cavity as well as the formation of neomembranes and their thicknesses were visible on endoscopy in the single burr-hole craniostomy treated patients. Out of the 40 
patients in whom endoscopy showed hematoma cavity separation or neomembrane thickness, hematoma recurred in 6 of them. We observed thickened membranes connecting/separating the dura and the thickened arachnoid/pia matters in the 6 patients with hematoma recurrence on endoscopy. These thickened membranes connecting/separating the dura and the thickened arachnoid/pia matters were often resected during endoscopy. Comparatively, endoscopy showed hematoma cavity separation or neomembrane thickness just as seen during craniotomy. Thus, we postulate that, inadequate or nonseparation of thickened membranes connecting/separating the dura and the thickened arachnoid/pia matters could be a cause of the recurrence of hematoma.

Mobbs et al suggested that, in certain peculiar cases, the endoscopy could be used to assist in recognizing neomembranes intra-operatively, as well as perhaps aid in its treatment either via endoscopyguided obliteration of the neomembrane or conversion to a normal craniotomy[18].

Chen et al indicated that, pre-operative CT scans showing inner subdural membranes may guide one to target the treatment to allow for the release of this inner subdural membranes and that, mini craniotomy with careful fenestration of the inner membrane is very effective treatment[45]. Their study did not use endoscopy to visualize as well as separate these inner membranes. Endoscopy is an accurate modality for both diagnosis as well as therapy.

Corticosteroids as well as atorvastatin have also been used in the management of $\operatorname{CSDH}[1,46,47]$. Nevertheless, surgical treatment is the most preferred treatment option of CSDH particularly in cases that present with mass effect[1]. Studies have shown that, morbidity as well as mortality of CSDH ranges from $0-25 \%$ and $0-32 \%$ respectively $[11,39,40]$. We observed mortality rate of $1.39 \%$. In all the deceased patients' treatment of the CSDH was not the direct cause of deaths. Our study is very limited because of the sample size and the number of recurrences. Thus, larger sample sizes will be needed to make definitive conclusions.

\section{Conclusion}

CT scan is capable of detecting of hematoma cavity separations and neomembrane thicknesses. On CT scan, neomembrane thicknesses were seen as abnormal structures relative to normal dura while hematoma cavity separations were seen as two or more subdural cavities. The treatment of patients with $\mathrm{CSDH}$ ought to include the identification and resection of abnormal thickened membranes connecting/separating the dura and the thickened arachnoid/pia matters to avoid recurrence.

Comparatively, endoscopy showed hematoma cavity separation or neomembrane thickness just as seen during craniotomy.

\section{Abbreviations}

$\mathrm{Cl}$

Confidence interval 
CSDH

Chronic subdural hematoma

CT

Computed tomography

$\mathrm{HU}$

Hounsfield Unit

OR

Odds Ratio

\section{Declarations}

Acknowledgements: We offer a special thanks to the Radiological Department of Zi Yang first people's hospital for their help on the radiological data collection and parameters measurement.

Funding Source: This study was supported by The Science and Technology Achievements Transfer and Transformation Project of Ziyang City (2018CZ-S-06).

Availability of Data: The data used in this paper is not publicly available but will be made available on reasonable demand from the corresponding author because of patients' confidentiality.

Author contributions: Study concepts and design: HL, RY, FX and SAR; Data acquisition and follow-up: HL, RY, FX and SAR; Statistical analysis: HL, RY, FX and SAR; Manuscript

preparation: SAR; Manuscript editing HL, RY, FX and SAR. All authors carefully reviewed the manuscript and approved the final version.

\section{Declarations}

Ethics approval and consent to participate: This study was approved by the Ethic Committee of Zi Yang first people's hospital and written informed consent was obtained from all participants. All methods were performed in accordance with the relevant guidelines and regulations.

Consent for publication: Informed consent for publication was obtained from all patients as well as their relatives.

Competing interests: All the authors have no competing interest to disclose.

\section{References}

1. Liu LX, Cao XD, Ren YM, Zhou LX, Yang CH: Risk Factors for Recurrence of Chronic Subdural Hematoma: A Single Center Experience. World Neurosurg 2019, 132:e506-e513.

2. Miranda LB, Braxton E, Hobbs J, Quigley MR: Chronic subdural hematoma in the elderly: not a benign disease. J Neurosurg 2011, 114(1):72-76. 
3. Maurice-Williams RS: Chronic subdural haematoma: an everyday problem for the neurosurgeon. $\mathrm{Br} \mathrm{J}$ Neurosurg 1999, 13(6):547-549.

4. Yang W, Huang J: Chronic Subdural Hematoma: Epidemiology and Natural History. Neurosurg Clin N Am 2017, 28(2):205-210.

5. Miah IP, Tank Y, Rosendaal FR, Peul WC, Dammers R, Lingsma HF, den Hertog HM, Jellema K, van der Gaag NA: Radiological prognostic factors of chronic subdural hematoma recurrence: a systematic review and meta-analysis. Neuroradiology 2021, 63(1):27-40.

6. Trotter W: Chronic subdural haemorrhage of traumatic origin, and its relation to pachymeningitis haemorrhagica interna. Br J Surg 1914, 2(6):271-291.

7. Drapkin AJ: Chronic subdural hematoma: pathophysiological basis for treatment. Br J Neurosurg 1991, 5(5):467-473.

8. Shim YS, Park CO, Hyun DK, Park HC, Yoon SH: What are the causative factors for a slow, progressive enlargement of a chronic subdural hematoma? Yonsei Med J 2007, 48(2):210-217.

9. Sahyouni R, Goshtasbi K, Mahmoodi A, Tran DK, Chen JW: Chronic Subdural Hematoma: A Historical and Clinical Perspective. World Neurosurg 2017, 108:948-953.

10. Torihashi K, Sadamasa N, Yoshida K, Narumi O, Chin M, Yamagata S: Independent predictors for recurrence of chronic subdural hematoma: a review of 343 consecutive surgical cases. Neurosurgery 2008, 63(6):1125-1129; discussion 1129.

11. Májovský M, Masopust V, Netuka D, Beneš V: Flexible endoscope-assisted evacuation of chronic subdural hematomas. Acta Neurochir (Wien) 2016, 158(10):1987-1992.

12. Cenic A, Bhandari M, Reddy K: Management of chronic subdural hematoma: a national survey and literature review. Can J Neurol Sci 2005, 32(4):501-506.

13. Mehta V, Harward SC, Sankey EW, Nayar G, Codd PJ: Evidence based diagnosis and management of chronic subdural hematoma: A review of the literature. J Clin Neurosci 2018, 50:7-15.

14. Weigel R, Schmiedek P, Krauss JK: Outcome of contemporary surgery for chronic subdural haematoma: evidence based review. Journal of neurology, neurosurgery, and psychiatry 2003 , 74(7):937-943.

15. Santarius T, Kirkpatrick PJ, Ganesan D, Chia HL, Jalloh I, Smielewski P, Richards HK, Marcus H, Parker $\mathrm{RA}$, Price SJ et al: Use of drains versus no drains after burr-hole evacuation of chronic subdural haematoma: a randomised controlled trial. Lancet 2009, 374(9695):1067-1073.

16. Kirollos RW, Tyagi AK, Boles DM: Endoscopy-assisted burr hole evacuation of subdural empyema. Br J Neurosurg 1996, 10(4):395-397.

17. Katsuki M, Kakizawa Y, Wada N, Yamamoto Y, Uchiyama T, Nakamura T, Watanabe M: Endoscopically Observed Outer Membrane Color of Chronic Subdural Hematoma and Histopathological Staging: White as a Risk Factor for Recurrence. Neurol Med Chir (Tokyo) 2020, 60(3):126-135. 
18. Mobbs R, Khong P: Endoscopic-assisted evacuation of subdural collections. J Clin Neurosci 2009, 16(5):701-704.

19. Nakaguchi $H$, Tanishima $T$, Yoshimasu N: Relationship between drainage catheter location and postoperative recurrence of chronic subdural hematoma after burr-hole irrigation and closed-system drainage. J Neurosurg 2000, 93(5):791-795.

20. Boyaci S, Gumustas OG, Korkmaz S, Aksoy K: Endoscopic Evacuation of Subdural Collections. Turk Neurosurg 2016, 26(6):871-877.

21. Kayaci S, Kanat A, Koksal V, Ozdemir B: Effect of inner membrane tearing in the treatment of adult chronic subdural hematoma: a comparative study. Neurol Med Chir (Tokyo) 2014, 54(5):363-373.

22. Cai Q, Guo Q, Zhang F, Sun D, Zhang W, Ji B, Chen Z, Mao S: Evacuation of chronic and subacute subdural hematoma via transcranial neuroendoscopic approach. Neuropsychiatr Dis Treat 2019, 15:385-390.

23. Zhang J, Liu X, Fan X, Fu K, Xu C, Hu Q, Jiang P, Chen J, Wang W: The use of endoscopic-assisted burr-hole craniostomy for septated chronic subdural haematoma: A retrospective cohort comparison study. Brain Res 2018, 1678:245-253.

24. Kim SU, Lee DH, Kim YI, Yang SH, Sung JH, Cho CB: Predictive Factors for Recurrence after Burr-Hole Craniostomy of Chronic Subdural Hematoma. J Korean Neurosurg Soc 2017, 60(6):701-709.

25. Oishi M, Toyama M, Tamatani S, Kitazawa T, Saito M: Clinical factors of recurrent chronic subdural hematoma. Neurol Med Chir (Tokyo) 2001, 41(8):382-386.

26. Matsumoto H, Hanayama H, Okada T, Sakurai Y, Minami H, Masuda A, Tominaga S, Miyaji K, Yamaura I, Yoshida Y: Which surgical procedure is effective for refractory chronic subdural hematoma? Analysis of our surgical procedures and literature review. J Clin Neurosci 2018, 49:4047.

27. Asghar M, Adhiyaman V, Greenway MW, Bhowmick BK, Bates A: Chronic subdural haematoma in the elderly-a North Wales experience. J R Soc Med 2002, 95(6):290-292.

28. Kudo H, Kuwamura K, Izawa I, Sawa H, Tamaki N: Chronic subdural hematoma in elderly people: present status on Awaji Island and epidemiological prospect. Neurol Med Chir (Tokyo) 1992, 32(4):207-209.

29. Shen J, Yuan L, Ge R, Wang Q, Zhou W, Jiang XC, Shao X: Clinical and radiological factors predicting recurrence of chronic subdural hematoma: A retrospective cohort study. Injury 2019, 50(10):16341640.

30. Altaf I, Shams S, Vohra AH: Radiolological predictors of recurrence of chronic subdural hematoma. Pak J Med Sci 2018, 34(1):194-197.

31. You W, Zhu Y, Wang Y, Liu W, Wang H, Wen L, Yang X: Prevalence of and risk factors for recurrence of chronic subdural hematoma. Acta Neurochir (Wien) 2018, 160(5):893-899.

32. Jang KM, Choi HH, Mun HY, Nam TK, Park YS, Kwon JT: Critical Depressed Brain Volume Influences the Recurrence of Chronic Subdural Hematoma after Surgical Evacuation. Sci Rep 2020, 10(1):1145. 
33. D'Abbondanza JA, Loch Macdonald R: Experimental models of chronic subdural hematoma. Neurol Res 2014, 36(2):176-188.

34. Frati A, Salvati M, Mainiero F, Ippoliti F, Rocchi G, Raco A, Caroli E, Cantore G, Delfini R: Inflammation markers and risk factors for recurrence in 35 patients with a posttraumatic chronic subdural hematoma: a prospective study. J Neurosurg 2004, 100(1):24-32.

35. Kitazono M, Yokota H, Satoh H, Onda H, Matsumoto G, Fuse A, Teramoto A: Measurement of inflammatory cytokines and thrombomodulin in chronic subdural hematoma. Neurol Med Chir (Tokyo) 2012, 52(11):810-815.

36. Nakamura S, Tsubokawa T: Extraction of angiogenesis factor from chronic subdural haematomas. Significance in capsule formation and haematoma growth. Brain Inj 1989, 3(2):129-136.

37. Tanaka Y, Ohno K: Chronic subdural hematoma - an up-to-date concept. J Med Dent Sci 2013, 60(2):55-61.

38. Richard SA, Wu M, Lin D: Traumatic Subdural Effusion Evolving into Chronic Subdural Hematoma. Open Journal of Modern Neurosurgery 2014, 5(01):12.

39. Brodbelt A, Warnke P: Outcome of contemporary surgery for chronic subdural haematoma: evidence based review. Journal of neurology, neurosurgery, and psychiatry 2004, 75(8):1209-1210; author reply 1210.

40. Ducruet AF, Grobelny BT, Zacharia BE, Hickman ZL, DeRosa PL, Andersen KN, Sussman E, Carpenter A, Connolly ES, Jr.: The surgical management of chronic subdural hematoma. Neurosurg Rev 2012, 35(2):155-169; discussion 169.

41. Chihara H, Imamura H, Ogura T, Adachi H, Imai Y, Sakai N: Recurrence of a Refractory Chronic Subdural Hematoma after Middle Meningeal Artery Embolization That Required Craniotomy. NMC Case Rep J 2014, 1(1):1-5.

42. Nakaguchi $\mathrm{H}$, Tanishima T, Yoshimasu $\mathrm{N}$ : Factors in the natural history of chronic subdural hematomas that influence their postoperative recurrence. J Neurosurg 2001, 95(2):256-262.

43. Hellwig D, Bauer BL: Minimally invasive neurosurgery by means of ultrathin endoscopes. Acta Neurochir Suppl (Wien) 1992, 54:63-68.

44. Karakhan VB, Khodnevich AA: Endoscopic surgery of traumatic intracranial haemorrhages. Acta Neurochir Suppl 1994, 61:84-91.

45. Chen JW, Xu JC, Malkasian D, Perez-Rosendahl MA, Tran DK: The Mini-Craniotomy for cSDH Revisited: New Perspectives. Front Neurol 2021, 12:660885.

46. Jiang R, Zhao S, Wang R, Feng H, Zhang J, Li X, Mao Y, Yuan X, Fei Z, Zhao Y et al: Safety and Efficacy of Atorvastatin for Chronic Subdural Hematoma in Chinese Patients: A Randomized ClinicalTrial. JAMA Neurol 2018, 75(11):1338-1346.

47. Berghauser Pont LM, Dirven CM, Dippel DW, Verweij BH, Dammers R: The role of corticosteroids in the management of chronic subdural hematoma: a systematic review. Eur J Neurol 2012, 19(11):1397-1403. 


\section{Figures}
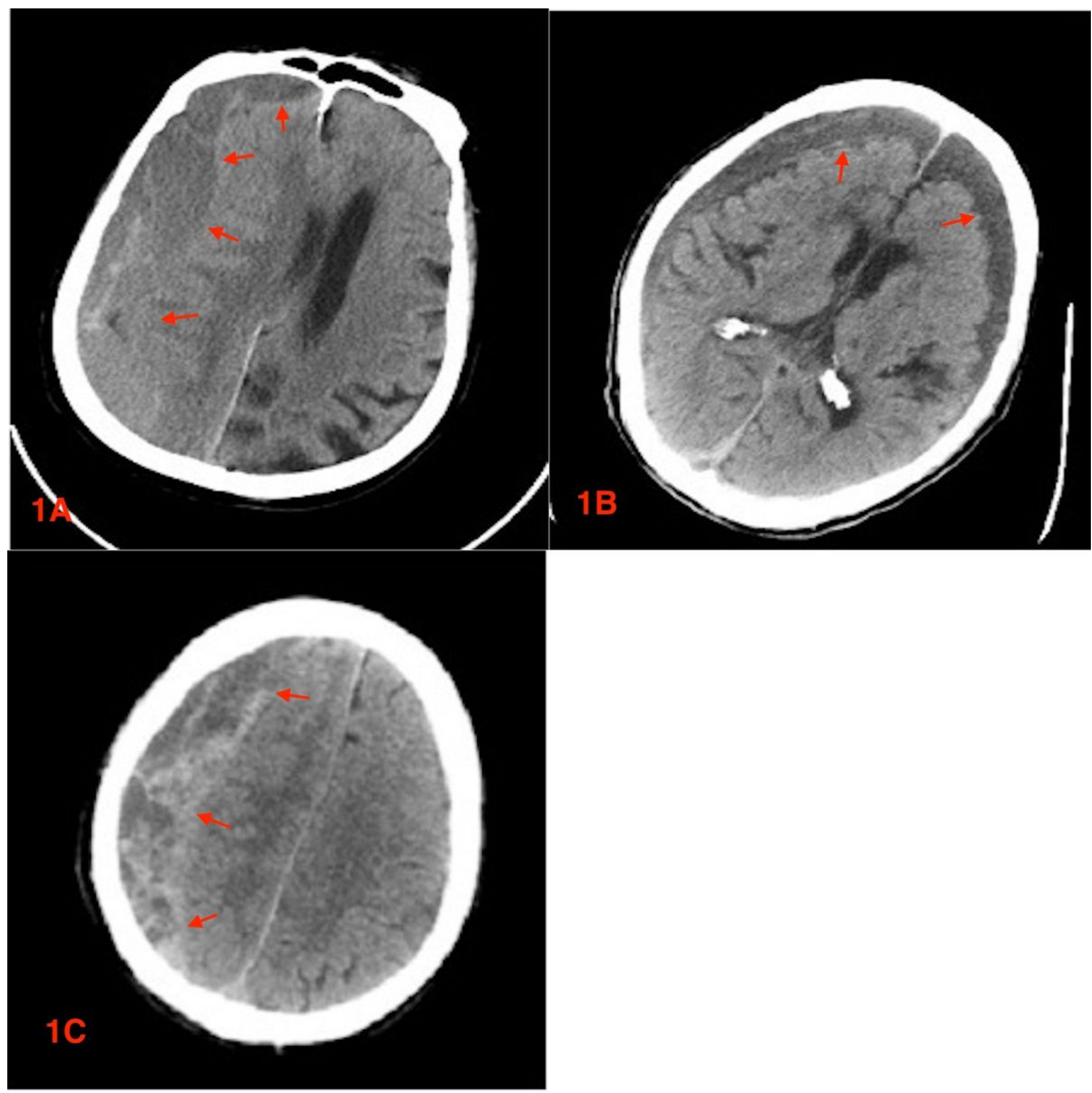

Figure 1

CT characteristic of patients: Hematoma cavity separation, new neomembrane and mixed density hematoma in CT. 1A \& B; hematoma cavity separation, $1 \mathrm{C}$; mixed density hematoma. Red arrow= boundaries of the abnormality 

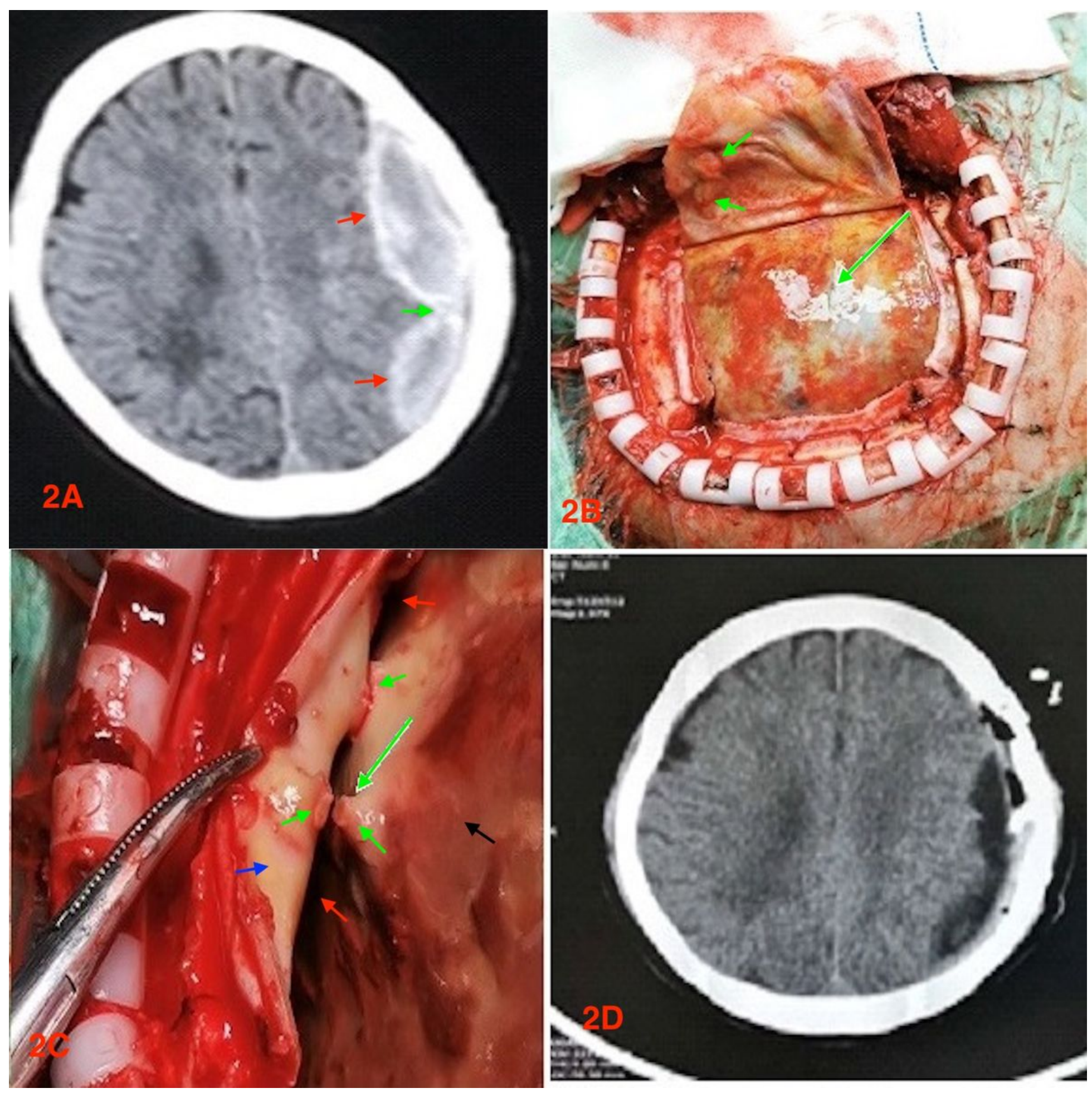

Figure 2

Preoperative CT scan, intraoperative images and postoperative CT scan on one of the patients without completely liquefied hematoma and hematoma cavity separation who underwent craniotomy.

2A: preoperative CT image: $R$ Red arrow = None liquefied hematoma; Green arrow = thickened membranes connecting/separating the dura and the thickened arachnoid/pia matters. 
2B\&C: Intraoperative images showing neomembrane, hematoma cavity separation as well as thickened membranes connecting/separating the dura and the thickened arachnoid/pia matters. 2B: Green arrows = thickened dura separators. 2C: Green arrows = thickened membrane connecting/separating the dura and the thickened arachnoid/pia matters, Red arrows = hematoma cavity separation, Black arrow= neomembrane and Blue arrow $=$ dura.

2D: Postoperative CT scan showing evaluation of the hematoma and separation of the thickened membranes connecting/separating the dura and the thickened arachnoid/pia matters.
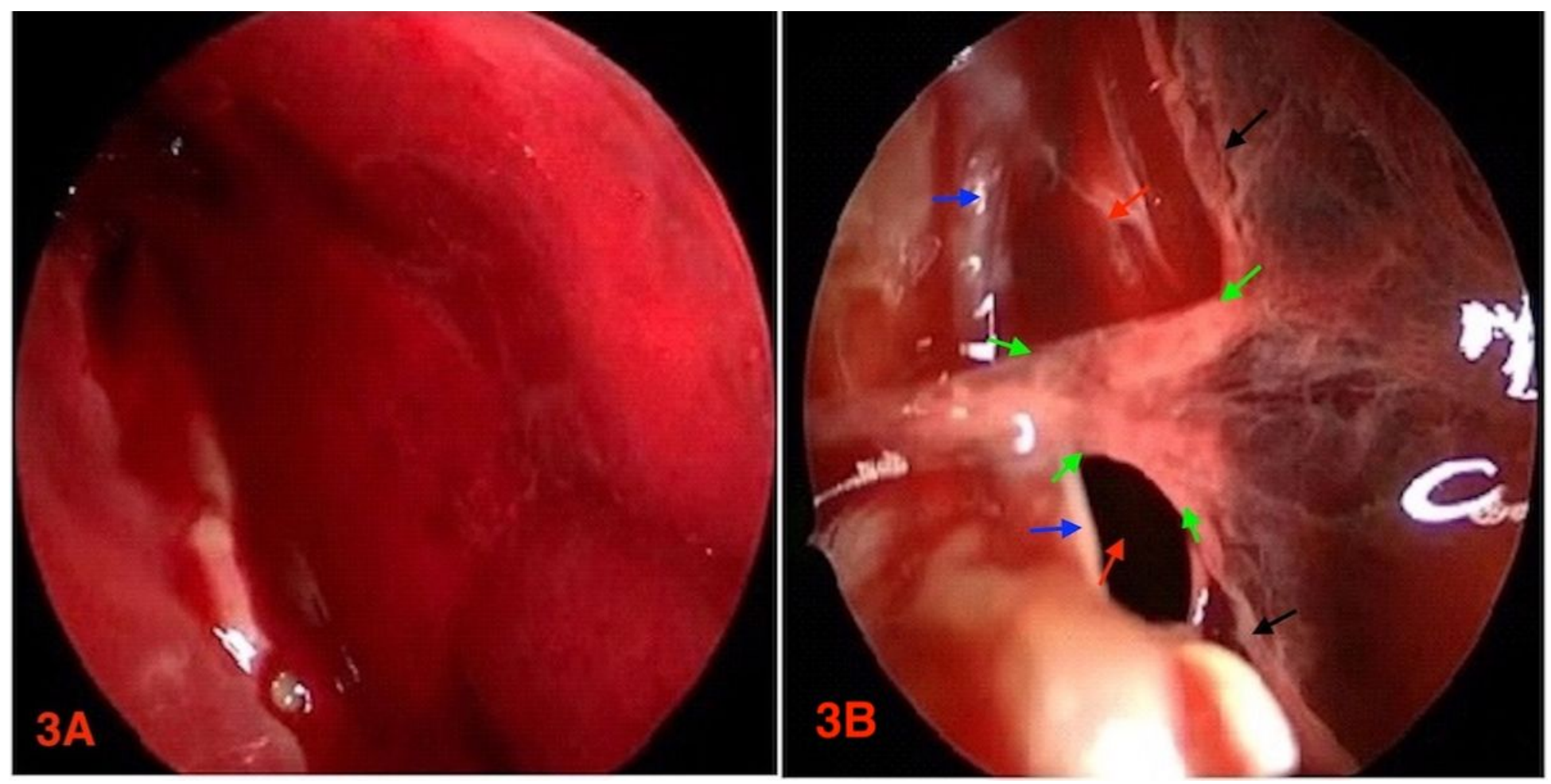

\section{Figure 3}

Intraoperative images showing the neomembrane, hematoma cavity separation in endoscopy as well as thickened membranes connecting/separating the dura and the thickened arachnoid/pia matters.

3A: Endoscopic image showing the neomembrane.

3B: Endoscopic image showing neomembrane, hematoma cavity separation as well as thickened membranes connecting/separating the dura and the thickened arachnoid/pia matters.

Black arrows = neomembrane, Red arrows = hematoma cavity separation, Blue arrows = dura and Green arrows $=$ thickened membranes connecting/separating the dura and the thickened arachnoid/pia matters. 